\title{
Balance, Basic Anthropometrics and Performance in Young Alpine Skiers; Longitudinal Analysis of the Associations During Two Competitive Seasons
}

\author{
by \\ Blaz Lesnik', Damir Sekulic ${ }^{2,3}$, Matej Supej ${ }^{1}$, Michael R Esco , Milan Zvan ${ }^{1}$
}

Balance is hypothesized to be important in alpine skiing, while it is known that balance depends on anthropometric indices. The aim of this investigation was to explore the association between balance, anthropometrics and skiing-results over two competitive seasons among youth alpine-skiers. Eighty-one skiers (40 females) participated in this study. The participants were tested twice over two competitive seasons: when they were 12-13 years old (U14) and when they were 14-15 years old (U16). The variables consisted of anthropometrics (body height and body mass) and three balance indexes (medio-lateral-, anterio-posterior-and overall-stability-index). Additionally, skiing results in U14 and U16 were evaluated. The balance status did not change significantly over the observed period of time regardless of the significant changes in body mass and height. The relationships between balance and skiing results were higher in the U14 (Pearson's $r=0.45-0.54$ ) than in the U16 (Pearson's $r=0.05-0.28$ ). The relationships between anthropometrics and competitive results were generally stronger in girls (Pearson's $r=0.39-0.88$ ) than in boys (Pearson's $r=0.26-0.58)$. After clustering athletes into three achievement groups on a basis of their competitive performance, discriminant canonical analysis showed that relationships between balance and skiing results decreased, while the relationships between anthropometrics and skiing results increased over the two observed seasons. This study highlighted the importance of balance in youth alpine skiing in the age range of 11-14 years.

Key words: coaching, testing, biomechanics, physical performance.

\section{Introduction}

Balance can be described as the process of maintaining the position of the body's center of gravity vertically over the base of support (Hrysomallis, 2011). It is generally accepted that balance is one of the most important motor abilities in alpine skiing (Hrysomallis, 2011; Hydren et al., 2013a, 2013b). This is additionally emphasized knowing that alpine skiing is a highintensity intermittent activity that results in lactate accumulation, muscle acidosis, peripheral neuromuscular fatigue, that altogether consequently influence proprioceptive deterioration (i.e. alter balance ability) (Douris et al., 2011; Ju et al., 2010; White and Wells, 2015).

Meanwhile, observations on the importance of balance in skiing are rarely directly confirmed throughout experimental settings. While there is an evident lack of research on this topic, studies performed previously have not confirmed the relationship between balance and skiing results (i.e. achievement, quality) (Males et al., 2013; Noe and Paillard, 2005; Platzer et al., 2009). Briefly, more successful alpine-skiers did not have superior balance when compared to their

1 - University of Ljubljana, Faculty of Sport, Ljubljana, Slovenia.

2 - University of Split, Faculty of Kinesiology, Split, Croatia.

3 - University of Split, University Department of Health Care Studies, Split, Croatia.

4 - University of Alabama, Department of Kinesiology, Tuscaloosa, AL, USA. 
less successful peers (Noe and Paillard, 2005). In addition, balance was not related to characteristic efficacy of skiing instructors (Males et al., 2013) or results of snowboarders (Platzer et al., 2009).

There are several possible explanations for the lack of research in this area. First, measurement of balance ability is difficult and unreliable out of laboratory settings as it requires relatively robust and expensive equipment (Sekulic et al., 2013). Second, results in alpine skiing are difficult to evaluate through a single competition (Hebert-Losier et al., 2014; Turnbull et al., 2009).

Another issue deserves attention. Namely, it is possible that balance ability is an important factor of sport-orientation and sport-selection for alpine skiing (i.e. similar to importance of body height in basketball), but not related to sport results at advanced competitive levels. This is possibly because the athletes who lack balance quit skiing due to being unsuccessful or that alpine skiing improves balance ability to the level necessary for successful competition. While balance is known to be strongly influenced by anthropometric features, it is also possible that developmental changes of the body build modulate the relationship between balance and skiing results to some extent (Claessens et al., 1999).

The aim of this study was to explore the relationship between balance ability and skiing performance among young alpine-skiers. Namely, the study examined skiing competition results and their relationship with balance ability and anthropometric variables over two competitive seasons. Additionally, the relationships between the main anthropometric features (body height and mass) and balance ability were examined.

\section{Material and Methods}

\section{Participants}

The sample of participants included eighty-one youth alpine-skiers (40 females) from Slovenia. At baseline, the athletes were involved in alpine skiing training for more than four years. All of the athletes were national competitors at the highest rank while many of them competed in various international events. They were invited to participate by the Ski Association of Slovenia. The Ethical Board of the University of Ljubljana, Faculty of Sport, provided approval of the research experiment. All subjects voluntarily participated in testing after being informed of the purpose of the study and after obtaining parental written consent. As a certain methodological remark, we must emphasize that athletes observed in this study were not specifically oriented toward speed and/or technical events, and all skiers observed herein competed in Slalom, Giant-slalom and Super G, while they were officially not included in Downhill events.

\section{Design}

This study was carried out in combined laboratory and field settings. Mainly, youth alpine skiers from Slovenia were observed over two competitive seasons. During the first observed season, which was considered baseline, the participants competed at the U14 level and were aged between 12 and 13 years. During the second observed season, they were between ages of 14 and 15 years and competed at the U16 level. The experimental approach consisted of several phases. Throughout the first phase, competitors were tested on independent variables at the beginning of the U14 season. The second phase comprised their alpine skiing competitions over the U14 season. The third phase included balance and anthropometric testing at the beginning of the U16 season, while the fourth phase comprised competing over the U16 season.

\section{Variables}

Testing comprised measuring anthropometric variables and balance ability. Anthropometric measures included body height and body mass evaluated by SECA equipment (Seca Instruments Ltd, Hamburg, Germany). Three balance indexes were recorded in this study as follows: medio-lateral balance index (MLI), anterio-posterior balance index (API), and overall stability index (OSI). Each variable was evaluated with a Biodex Balance System (Biodex Medical Systems, Shirley, NY, USA), which was a stabilometer where athletes were asked to maintain a stable position on an unstable platform (Sekulic et al., 2013). The apparatus measured the degrees of deviation from the ideal horizontal position. The MLI and API are indexes of an average tilt in each specific plane, while the OSI is an index that incorporates both the results of MLI and API. For all three indexes, a greater tilt from the ideal vertical position over the base of the support platform provides a higher numerical 
value which indicates greater instability. The athletes were instructed to establish a most convenient foot position and comfortable stance width that allowed them to maintain the most stable, horizontal position possible on the platform for the duration of the test. The positions were marked with tape using coordinates on the platform's grid to ensure the same stance was consistently maintained during each testing trial. The participants maintained an upright posture while their arms were kept by the side. The resistance level was set at number 4 on a scale ranging from 1 (least stable) to 12 (most stable). Balance testing was performed during three trials each lasting $20 \mathrm{~s}$, with 2-3 min rest between trials. The reliability was appropriate with the ICC ranging for 0.76-0.81 (for boys) and 0.79-0.81 (for girls), which was consistent with previous studies conducted on similar samples of participants (Sekulic et al., 2014). Also, repeated measures ANOVA calculated between testing trials found no significant effects, emphasizing no systematic bias (i.e. no learning effects).

Anthropometric measurements were carried out at the beginning of testing. Afterwards, the participants performed a $4-5 \mathrm{~min}$ warm up (half-squats, light jumps, lunges, etc.) and dynamic stretching. They were familiarized with balance testing equipment and completed 10 $\mathrm{s}$ pre-testing (not included in analyses). All participants were tested in the same order. To avoid diurnal variations, all tests were carried out in the morning from 8 to $11 \mathrm{am}$.

For the U14 and U16, the competitive performance (i.e. skiing result) was indicated through the final result at the highest nationallevel competition for observed ages (i.e. "Rauch Cup"), consisting of altogether ten races. The final result was condensed as a sum of all races.

\section{Statistics}

Kolmogorov-Smirnov tests revealed all variables as normally distributed. Therefore, statistics included means and standard deviations. The changes over the observed period (i.e. between U14 and U16) were identified by the dependent t-test. To define the relationships between variables, Pearson's correlations were calculated. Finally, the athletes were divided into achievement groups according to their competitive performance in each observed season. The low-achievement group comprised one-third of the athletes with the poorest results, the average-achievement group consisted of those athletes who were ranked between 33rd and 66th percentile, and the high-achievement group comprised one-third of the sample that displayed the most successful scores. Such grouping was done independently for the two observed competitive seasons. To define differences between the achievement-groups, a discriminant canonical analysis (DISCRA) was performed (Sattler et al., 2015). Since the OSI is a linear combination of the API and MLI, it was not included in the DISCRA calculation. All analyses were gender-stratified. Statistical significance was pre-determined as $p<0.05$. Statistica, ver. 11 (Statsoft, Tulsa, OK) was used for all statistical calculations.

\section{Results}

The observed anthropometric measures significantly changed for both boys and girls. However, their balance status did not change between the U14 and U16. Boys were significantly taller and heavier, while girls were advanced in balance abilities (Table 1).

The correlations between body height and body mass with the OSI in the U14 and U16 were significant for boys (Figure 1). Meanwhile, the correlation between body height and the OSI in U16 did not reach statistical significance for girls (Figure 2). Due to the opposite metrics (i.e. lower numerical values on balance indexes present better result), the positive correlations denote negative relationships between anthropometrics and balance.

For the U14 season, balance measures and body height were significantly correlated with competitive performance for boys. For the U16 season, body height and body mass were significantly correlated to skiing results for boys. Among the girls, all balance measures were significantly associated to the U14, but not to the U16 skiing results. The correlations between anthropometrics and competitive performance in U14 and U16 seasons for girls were significant (Table 2).

When correlations between anthropometric and balance indices with competitive performance were calculated separately for high-achievers, average-achievers and low-achievers, a certain trend of higher 
correlations between balance and competitive performance was observed in low-achievement groups (for boys and girls) (Table 3 ).

The discriminant analysis revealed significant differences between the achievementgroups based on U14 results for boys and girls. The MLI, body height and body mass contributed equally to U14 skiing performance among the boys, with best skiing performance in taller and heavier boys who possessed advanced MLI capacity. The API and MLI were equally important in defining the differences between U14 achievement groups among girls. In both girls and boys, balance was positively associated with competitive performance. The classification was correct for $81 \%$ of boys and $85 \%$ of girls (Table 4 ).

The most important determinants of the U16 results for the girls were body height and body mass. Those girls with higher body mass and body height were more likely to achieve better results. $76 \%$ of girls were correctly classified $(84 \%$ of low-achievers, $63 \%$ of average-achievers and $79 \%$ of high-achievers). The discriminant analysis did not reach statistical significance for the boys' U16 results (Table 4).

Table 1

Descriptive statistics and univariate differences between U14 and U16 competitive seasons (dependent samples t-test) and between genders (independent samples t-test)

\begin{tabular}{|lcccc|}
\hline & U14 & U16 & \multicolumn{2}{c|}{ T test } \\
& Mean \pm SD & Mean \pm SD & T value & $p$ \\
\cline { 2 - 5 } Boys & & & & \\
BM (kg) & $50.3 \pm 13.9$ & $58.9 \pm 15.2$ & -14.88 & 0.01 \\
BH (cm) & $160.5 \pm 12.1$ & $168.5 \pm 12.6$ & -14.53 & 0.01 \\
API (index) & $1.24 \pm 0.69$ & $1.27 \pm 0.64$ & -0.41 & 0.67 \\
MLI (index) & $1.05 \pm 0.58$ & $1.03 \pm 0.54$ & 0.21 & 0.83 \\
OSI (index) & $1.81 \pm 0.96$ & $1.84 \pm 0.89$ & -0.31 & 0.74 \\
Girls & & & & \\
BM (kg) & & & & \\
BH (cm) & $14.5 \pm 9.1^{*}$ & $52.3 \pm 8.9^{¥}$ & -17.01 & 0.01 \\
API (index) & $1.01 \pm 0.43^{*}$ & $1.04 \pm 0.54^{¥}$ & -0.72 & 0.48 \\
MLI (index) & $0.81 \pm 0.39^{*}$ & $0.77 \pm 0.33^{¥}$ & 0.98 & 0.32 \\
OSI (index) & $1.44 \pm 0.61^{*}$ & $1.44 \pm 0.63^{¥}$ & 0.13 & 0.89 \\
\hline
\end{tabular}

$B M-b o d y$ mass, $B H$ - body height, API - anterio-posterior balance index, $M L I$ - medio-lateral balance index, OSI - overall stability index (for balance indexes lower numerical result represents better achievement), * present significant $t$-test differences between genders for the U14, ${ }^{*}$ present significant $t$-test differences between genders for the U16 
Fig la. Body mass and OSI in U14

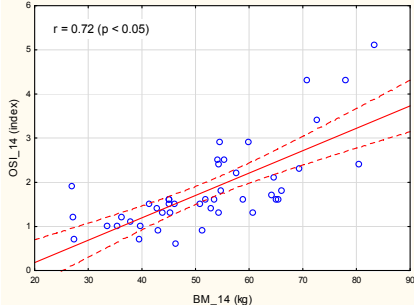

Fig 1c. Body mass and OSI in U16

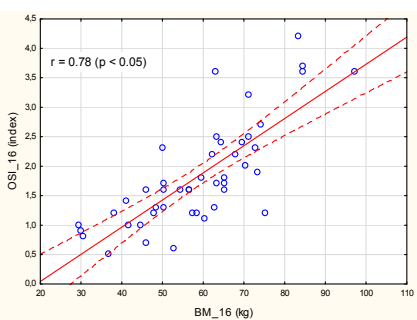

Fig 1b. Body height and OSI in U14

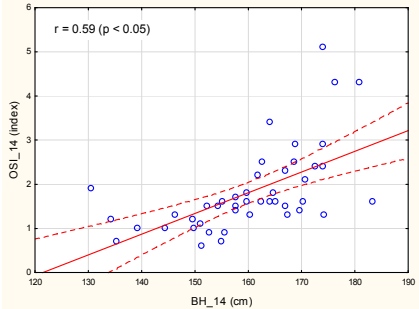

Fig 1d. Body height and OSI in U16

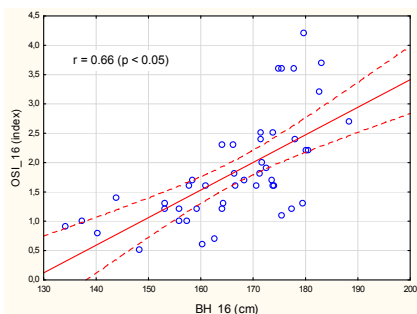

Figure 1

Correlations between anthropometrics (BH - body height, BM - body mass) and overall stability index (OSI) for boys

Fig 1a. Body mass and OSI in U14

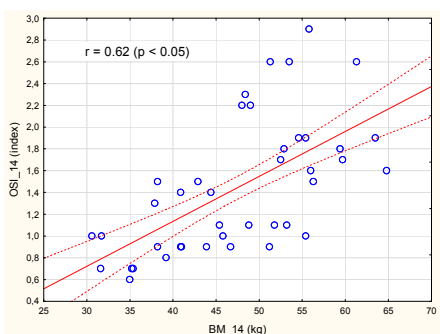

Fig 1c. Body mass and OSI in U16

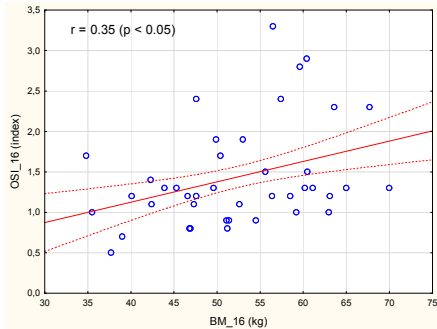

Fig 1 b. Body height and OSI in U14

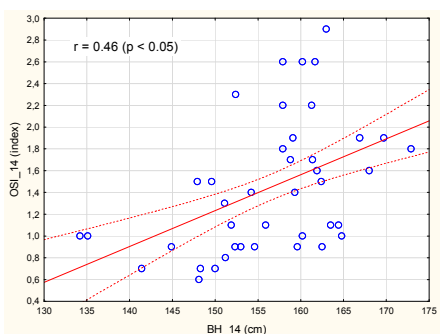

Fig 1d. Body height and OSI in U16

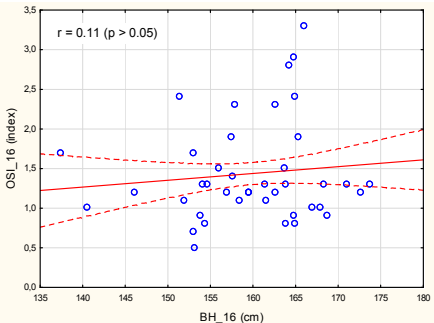

Figure 2

Correlations between anthropometrics (BH - body height, BM-body mass) and overall stability index (OSI) for girls 
Table 2

Pearson's correlation between observed variables for U14 and U16 seasons

\begin{tabular}{|c|c|c|c|c|c|c|}
\hline & \multicolumn{3}{|c|}{ Boys } & \multicolumn{3}{|c|}{ Girls } \\
\hline & API & MLI & $\begin{array}{c}\text { Competitive } \\
\text { Result }\end{array}$ & API & MLI & $\begin{array}{c}\text { Competitive } \\
\text { Result }\end{array}$ \\
\hline \multicolumn{7}{|l|}{ U14 } \\
\hline $\mathrm{BM}(\mathrm{kg})$ & $0.66^{*}$ & $0.74^{*}$ & -0.26 & $0.58^{*}$ & $0.64^{*}$ & $-0.39^{*}$ \\
\hline $\mathrm{BH}(\mathrm{cm})$ & $0.51^{*}$ & $0.64^{*}$ & $-0.42^{*}$ & $0.41^{*}$ & $0.48^{*}$ & $-0.41^{*}$ \\
\hline API (index) & - & $0.78^{*}$ & $0.54^{*}$ & - & - & $0.45^{*}$ \\
\hline MLI (index) & $0.78^{*}$ & - & $0.54^{*}$ & $0.77^{*}$ & - & $0.46^{*}$ \\
\hline OSI (index) & $0.96^{*}$ & $0.97^{*}$ & $0.53^{*}$ & $0.98^{*}$ & $0.97^{*}$ & $0.45^{*}$ \\
\hline \multicolumn{7}{|l|}{ U16 } \\
\hline $\mathrm{BM}(\mathrm{kg})$ & $0.72^{*}$ & $0.82^{*}$ & $-0.58^{*}$ & 0.39 & 0.25 & $-0.82^{*}$ \\
\hline $\mathrm{BH}(\mathrm{cm})$ & $0.61^{*}$ & $0.69^{*}$ & $-0.57^{*}$ & 0.10 & 0.14 & $-0.88^{*}$ \\
\hline API (index) & - & - & 0.05 & - & - & 0.28 \\
\hline MLI (index) & $0.74^{*}$ & - & 0.13 & $0.72^{*}$ & - & 0.24 \\
\hline OSI (index) & $0.98^{*}$ & $0.97^{*}$ & 0.03 & $0.95^{*}$ & $0.94^{*}$ & 0.26 \\
\hline
\end{tabular}

$B M-$ body mass, BH - body height, API - anterio-posterior balance index, MLImedio-lateral balance index, OSI - overall stability index (for balance indexes lower numerical result represents better achievement), * denotes significance of $p<0.05$

\section{Table 3}

Pearson's correlation between observed variables for the three achievement groups

\begin{tabular}{|lcccccc|}
\hline & \multicolumn{2}{c}{ U14 competitive result } & \multicolumn{4}{c|}{ U16 competitive result } \\
& HA & AA & LA & HA & AA & LA \\
\cline { 2 - 7 } Boys & & & & & & \\
BM (kg) & 0.36 & -0.26 & $-0.73^{*}$ & -0.23 & -0.28 & -0.56 \\
BH (cm) & -0.46 & -0.56 & -0.50 & $-0.65^{*}$ & $-0.68^{*}$ & $-0.92^{*}$ \\
API (index) & 0.30 & 0.54 & 0.48 & 0.11 & -0.08 & 0.30 \\
MLI (index & 0.38 & 0.53 & $0.58^{*}$ & 0.08 & -0.14 & 0.34 \\
OSI (index) & 0.27 & 0.53 & 0.53 & 0.10 & -0.12 & 0.33 \\
Girls & & & & & & \\
BM (kg) & -0.19 & -0.21 & -0.31 & $-0.78^{*}$ & $-0.67^{*}$ & $-0.67^{*}$ \\
BH (cm) & -0.10 & -0.19 & -0.23 & -0.50 & $-0.60^{*}$ & $-0.59^{*}$ \\
API (index) & 0.14 & 0.26 & 0.52 & 0.19 & 0.23 & 0.23 \\
MLI (index & 0.17 & 0.30 & 0.50 & 0.13 & 0.21 & 0.16 \\
OSI (index) & 0.15 & 0.28 & 0.50 & 0.16 & 0.22 & 0.18 \\
\hline
\end{tabular}

$B M$ - body mass, BH - body height, API - anterio-posterior balance index, $M L I-$ medio-lateral balance index, OSI - overall stability index (for balance indexes lower numerical result represents better achievement), $H A$-high achievers, $A A$-average achievers, $L A$ - low achievers, * denotes significance of $p<0.05$ 
Table 4

Multivariate differences (canonical discriminant analyses) between achievement groups formed on a basis of the skiing in U14 and U16 seasons

\begin{tabular}{|lccccccccc|}
\hline & \multicolumn{4}{c}{ Males } \\
& \multicolumn{3}{c}{$\mathrm{U} 14$} & \multicolumn{2}{c}{$\mathrm{U} 16$} & \multicolumn{3}{c}{ U14 } & U16 \\
\cline { 2 - 9 } & Root 1 & Root 2 & Root 1 & Root 2 & Root 1 & Root 2 & Root 1 & Root 2 \\
BM (kg) & 0.65 & 0.02 & 0.61 & 0.21 & 0.25 & 0.84 & -0.74 & 0.36 \\
BH (cm) & 0.61 & 0.07 & 0.72 & 0.11 & 0.18 & 0.84 & -0.71 & 0.45 \\
API (index) & 0.11 & 0.53 & 0.62 & 0.23 & -0.42 & 0.81 & 0.25 & -0.40 \\
MLI (index & -0.58 & 0.37 & 0.51 & -0.11 & -0.45 & 0.75 & 0.18 & 0.15 \\
Canonical R & 0.81 & 0.38 & 0.33 & 0.27 & 0.78 & 0.37 & 0.75 & 0.25 \\
Wilks Lambda & 0.52 & 0.78 & 0.84 & 0.92 & 0.58 & 0.81 & 0.59 & 0.93 \\
$p$ & 0.01 & 0.46 & 0.41 & 0.52 & 0.03 & 0.34 & 0.02 & 0.42 \\
Centroid: Low & -1.02 & -0.33 & -0.57 & 0.08 & -0.89 & -0.49 & 0.91 & -0.12 \\
achievers & & & & & & & & \\
Centroid: Average & 0.21 & 1.45 & 0.18 & -0.40 & 0.05 & 0.42 & 0.55 & 0.38 \\
achievers & & & & & & & & \\
Centroid: High & 1.12 & 0.07 & 0.37 & 0.28 & 1.11 & 0.09 & -0.47 & -0.21 \\
achievers & & & & & & & &
\end{tabular}

$B M$ - body mass, BH - body height, API - anterio-posterior balance index, $M L I-$ medio-lateral balance index (for balance indexes lower numerical result represents better achievement)

\section{Discussion}

There are several important findings of the study. First of all, the results suggest that balance status of youth skiers did not change significantly over the observed period of time regardless of the evident changes in anthropometric status. Second, the relationships between balance and anthropometrics were higher in boys than in girls and decreased during the course of the study. Finally, the relationship between balance and skiing performance decreased, while the relationships between anthropometrics and competitive performance increased over the two seasons.

The fact that the current study indicated that balance was maintained during the period of life when anthropometric measures significantly changed is highly indicative and supportive to previous investigations that reported skiing as an effective balance exercise (Wojtyczek et al., 2014). Postural balance is known to be more affected by anthropometrics in males than in females (Alonso et al., 2012). However, the current study is one of the first to report correlations between anthropometrics and balance ability in a longitudinal manner. Interestingly, the correlation between anthropometrics and balance was generally stronger in the U14 compared to the U16 season, which was consistent for both genders. Almost certainly, this finding is directly related to the adolescent growth spurt during the observed years (Malina et al., 2004; Ostojic et al., 2014).

Our results are somewhat contradictory to previous studies that have reported no significant relationships between balance and skiing 
performance (Males et al., 2013; Noe and Paillard, 2005; Platzer et al., 2009). Explaining the diverse findings, we must emphasize several differences in experimental designs of our compared to previous investigations. First, we examined youth skiers and used reliable and sophisticated balance measuring equipment throughout laboratory settings, while previous studies sampled adult ski-instructors using field testing protocols (Males et al., 2013), adult athletes (Noe and Paillard, 2005), and/or snowboarders (Platzer et al., 2009). Indeed, our results indicated balance as an important determinant of success only in younger age (U14), which almost certainly contributed to different findings. Second, in our study we observed a true competitive result as a criterion, and observed a relatively large number of participants. In the meantime, in one study that actually observed alpine skiers, balance abilities of seven national- and seven regional-level skiers were evaluated and compared, which almost certainly limited the possibility of reaching statistical significance of the results (Noe and Paillard, 2005). Herein, the correlations between balance ability and competitive performance for different achievement groups were relatively higher in low- and average-achievement skiers (Table 3), which additionally support the later explanation.

While the importance of overall balance in skiing is relatively known, the importance of different balance indices (mediolateral- and anteriopostural-balance) is rarely discussed. Specifically, one of the most crucial aspects of successful skiing is the ability to perform a sharp turn and resist the forces generated during the turn while maintaining edge control and balance (Hydren et al., 2013b). Meanwhile, for the purpose of achieving optimal pressure on ski-edges, the athlete must assure proper "cuff pressure". In other words, the shin must apply pressure to the front of the boot. At the same time, the upper body should remain stable without excessive movement of the arms. With the constant changes of velocity (acceleration), due to inertia, the skier's body tends to lean backward for positive acceleration or lean forward for negative acceleration. Consequently, the skier's ability to resist such perturbations is directly related to anterio-postural stability as measured by the API.

Medio-lateral balance (as measured by the
MLI) is challenged mostly during ski turns when the skier has to lean toward the center of the turn in order to compensate centripetal force (i.e. radial acceleration over the turn) (Hydren et al., 2013b). Since racing terrain (i.e. snow) is not ideally flat, balance is constantly disrupted. Therefore, it seems logical that the athlete's ability to maintain ideal balance determines final competitive performance to some extent. This is even more pronounced in the age of U14 because of the recent growth spurt and the negative influence of anthropometric changes on balance ability.

However, it is not only balance that contributes to U14 performance, but other factors as well. Namely, discriminant analysis revealed the importance of higher body mass and height in boys as the most superior skiing performance was shown in the boys who possessed superior balance while being relatively tall and heavy at the same time, which is characteristic of those children of advanced biological age (i.e. early developers) (Malina et al., 2004).

The need for better balance of skiers that are more advanced in growth may be explained through the following requirements and needs of alpine skiing. Using classical mechanics, it can be derived that relative ground reaction forces per unit of mass are equivalent at equal turn radii and skiing speed regardless of the body mass of the skier. However, the latter is not enough to understand the need for balance in skiing. Skiers who are taller and heavier have longer segments. Therefore, the joints produce greater torque at equal joint angles (i.e., with the same technique of skiing) (Zatsiorsky, 2002). The taller and heavier skiers should have more strength due to their body mass and the prolonged levers of torque. Since ground reaction forces and range of motion in the joints of competitive alpine skiing are rather large (Gilgien et al., 2013; Supej, 2010; Supej et al., 2004; Vaverka and Vodickova, 2010), high joint torque is also present (Supej, 2010).

\section{Study limitations}

This study included children undergoing growth and developmental changes while the exact level of those changes (i.e. biological age) was not evaluated. Not knowing the relationship between biological age and conditioning abilities is probably the main limitation of this investigation. Also, some potentially important predictors of skiing performance were not 
observed (i.e. strength and endurance). Finally, we studied only one aspect of balance (stability index), while some other determinants of balance ability were not investigated (i.e. limits of stability, dynamic balance). Therefore, in future studies the present limitations should be taken into account.

\section{Conclusion}

The strong relationship between anthropometrics and balance in the U14 confirmed the negative influence of dynamic growth and development during this age range (i.e. growth spurt) on balance.

The highest skiing performance at the age of 14-15 years should be expected for those skiers who have good balance while being relatively tall and heavy. While the positive influence of balance is clear due to the dynamic nature of skiing, the higher body mass and height probably indicate advanced maturation and consequent superior physical capacities of those children.

There are practical implications from this study that are relevant to the sport of skiing. This study indirectly defined the positive influence of alpine skiing training on balance improvement. Also, balance training should be considered as an important part of the strength and conditioning process in youth alpine skiing, especially at the age range of 11-14 years. Next, in improving balance, special attention should be paid to those children who experienced dynamic growth over the period of 11-13 years of age. Finally, there are certain indices that alpine skiing should be observed as a type of physical activity that may prevent chronological alterations in balance of pubertal children.

\section{References}

Alonso AC, Luna NM, Mochizuki L, Barbieri F, Santos S, Greve JM. The influence of anthropometric factors on postural balance: the relationship between body composition and posturographic measurements in young adults. Clinics, 2012; 67: 1433-1441

Claessens AL, Lefevre J, Beunen G, Malina RM. The contribution of anthropometric characteristics to performance scores in elite female gymnasts. J Sports Med Phys Fitness, 1999; 39: 355-360

Douris PC1, Handrakis JP, Gendy J, Salama M, Kwon D, Brooks R, Salama N, Southard V. Fatiguing upper body aerobic exercise impairs balance. J Strength Cond Res, 2011; 25: 3299-3305 doi: 10.1519/JSC.0b013e318215fa07

Gilgien M, Spörri J, Chardonnens J, Kröll J, Müller E. Determination of External Forces in Alpine Skiing Using a Differential Global Navigation Satellite System. Sensors, 2013; 13: 9821-9835

Hebert-Losier K, Supej M, Holmberg HC. Biomechanical factors influencing the performance of elite Alpine ski racers. Sports Med, 2014; 44: 519-533. doi: 10.1007/s40279-013-0132-z

Hrysomallis C. Balance Ability and Athletic Performance. Sports Med, 2011; 41: 221-232 doi: 10.2165/11538560-000000000-00000

Hydren JR, Kraemer WJ, Volek JS, Dunn-Lewis C, Comstock BA, Szivak TK, Hooper DR, Denegar CR, Maresh CM. Performance changes during a weeklong high-altitude alpine ski-racing training camp in lowlander young athletes. J Strength Cond Res, 2013; 27: 924-937 doi: 10.1519/JSC.0b013e31827a9c62

Hydren JR, Volek JS, Maresh CM, Comstock BA, Kraemer WJ. Review of Strength and Conditioning for Alpine Ski Racing. Strength Cond J, 2013; 35: 10-28 doi: 10.1519/SSC.0b013e31828238be

Ju YY, Wang CW, Cheng HY. Effects of active fatiguing movement versus passive repetitive movement on knee proprioception. Clin Biomech, 2010; 25: 708-712 doi: 10.1016/j.clinbiomech.2010.04.017

Males B, Franjko I, Kuna D. Relations of Biomotor Structures and Performance of Technical Elements of Alpine Skiing in Croatian Ski Instructors. Coll Anthropol, 2013; 37: 77-82

Malina RM, Bouchard C, Bar-Or O. Growth, Maturation, and Physical Activity. Champaign: Human Kinetics; 2004. 
Noe F, Paillard T. Is postural control affected by expertise in alpine skiing? Br J Sports Med, 2005; 39: 835-837 doi: 10.1136/bjsm.2005.018127

Ostojic SM, Castagna C, Calleja-Gonzalez J, Jukic I, Idrizovic K, Stojanovic M. The biological age of 14-yearold boys and success in adult soccer: do early maturers predominate in the top-level game? Res Sports Med, 2014; 22: 398-407. doi: 10.1080/15438627.2014.944303

Platzer HP, Raschner C, Patterson C, Lembert S. Comparison of physical characteristics and performance among elite snowboarders. J Strength Cond Res, 2009; 23: 1427-1432 doi: 10.1519/JSC.0b013e3181aa1d9f

Sattler T, Sekulic D, Spasic M, Peric M, Krolo A, Uljevic O, Kondric M. Analysis of the Association Between Motor and Anthropometric Variables with Change of Direction Speed and Reactive Agility Performance. J Hum Kinet, 2015; 47: 137-145 doi: 10.1515/hukin-2015-0069

Sekulic D, Spasic M, Esco MR. Predicting agility performance with other performance variables in pubescent boys: a multiple-regression approach. Percept Mot Skills, 2014; 118: 447-461 doi: 10.2466/25.10.PMS.118k16w4

Sekulic D, Spasic M, Mirkov D, Cavar M, Sattler T. Gender-specific influences of balance, speed, and power on agility performance. J Strength Cond Res, 2013; 27: 802-811 doi: 10.1519/JSC.0b013e31825c2cb0

Supej M, Kugovnik O, Nemec B. Modelling and simulation of two competition slalom techniques. Kinesiology, 2004; 36: 206-212

Supej M. 3D measurements of alpine skiing with an inertial sensor motion capture suit and GNSS RTK system. J Sports Sci, 2010; 28: 759-769

Turnbull JR, Kilding AE, Keogh JWL. Physiology of alpine skiing. Scand J Med Sci Sports, 2009; 19: 146-155 doi: 10.1111/j.1600-0838.2009.00901.x

Vaverka F, Vodickova S. Laterality of the lower limbs and carving turn. Biol Sport, 2010; 27: 129-134

White GE, Wells GD. The effect of on-hill active recovery performed between runs on blood lactate concentration and fatigue in alpine ski racers. J Strength Cond Res, 2015; 29: 800-806 doi: 10.1519/JSC.0000000000000677

Wojtyczek B, Paslawska M, Raschner C. Changes in the Balance Performance of Polish Recreational Skiers after Seven Days of Alpine Skiing. J Hum Kinet, 2014; 44: 29-40 doi: 10.2478/hukin-2014-0108

Zatsiorsky VM. Kinetics of Human Motion. Champaign: Human Kinetics; 2002.

\section{Corresponding author:}

\section{Damir Sekulic,}

University of Split, Faculty of Kinesiology,

Teslina 6, Split - 21000, Croatia

phone/fax: + 38521302440

e-mail: dado@kifst.hr 